\title{
A Study of Ankle- Brachial Index In Patients Of Stroke
}

\author{
Dr. Neha Sharma ${ }^{1}$,Dr. Alok Gupta ${ }^{2}$,Dr. Priyanka P. ${ }^{3}$, Dr. Rajpal Singh ${ }^{4}$,Dr. Rajat \\ Gupta $^{5}$,Dr. Deepak Sharma ${ }^{6}$ \\ ${ }^{1}$ (Senior Resident, Dept. Of General Medicine, Dr. S.N.M.C. Jodhpur, India) \\ ${ }_{2}^{2}$ (Senior Professor, Dept. Of General Medicine, Dr. S.N.M.C. Jodhpur, India) \\ ${ }^{3}$ (Senior Resident, Dept. Of General Medicine, Dr. S.N.M.C. Jodhpur, India) \\ ${ }_{5}^{4}$ (Resident, Dept. Of General Medicine, Dr. S.N.M.C. Jodhpur, India) \\ ${ }^{5}$ (Resident, Dept. Of General Medicine, Dr. S.N.M.C. Jodhpur, India) \\ ${ }^{6}$ (Assistant Professor, Dept. Of General Medicine, Dr. S.N.M.C. Jodhpur, India)
}

\begin{abstract}
Stroke is the third leading cause of morbidity and mortality all over the world. ${ }^{[1]}$ In both primary and secondary prevention of stroke, Peripheral Arterial Disease (PAD) indicates a high risk of future events and plays an important role in stroke rehabilitation. The basic pathology behind macrovascular diseases is atherosclerosis, thus its early detection with noninvasive techniques such as ankle brachial index (ABI) measurement can be pivotal .A prospective observational cross sectional study was carried out in 50 patients with clinical \& imaging based evidence of stroke over 6 months at Mahatma Gandhi Hospital, Jodhpur. All patients with conditions, which affect ABI measurement,were excluded. ABI was calculated by measuring systolic BP in ankle, brachial vessels located using hand held Doppler device. Clinical and radiological assessment with detailed history was carried out. Data obtained was statistically analyzed. Out of 50 stroke patients included, 41 had low ABI indicating PAD. Among them 42\% had moderate PAD, 8\% had severe PAD and $18 \%$ had normal ABI. $24 \%$ of patients had past CVA, 16\% had previous IHD. Mean systolic BP in upper and lower limb was significantly different. Correlation between $A B I$ and stroke was statistically significant, more with ischemic type. ABI significantly correlated with systolic BP of lower limb and difference of SBP in upper \& lower limb at each side. A difference of >10mm $\mathrm{Hg}$ in SBP of both upper limbs was found in 23 patients of which 22 had low ABI. Patients with co-morbid conditions as HTN, DM, IHD, past CVA or with abnormal waist-Hip ratio, dyslipidemia, tobacco \& smoking addiction were more in low ABI group. We infer that patients of stroke harbor a major burden of atherosclerosis in their various vascular territories, early detection of which can help to determine the prognosis \& reduce the morbidity and mortality.
\end{abstract}

Keywords: stroke, ABI, PAD, atherosclerosis

\section{Introduction}

Stroke is one of the commonly encountered cases in Department of Medicine associated with significant morbidity and mortality. Stroke or cerebrovascular accident is defined as an abrupt onset neurological deficit attributable to a vascular cause. Complications occurring in rehabilitation period in stroke patients are important determinants of prognosis. Major ones are recurrence of stroke, other major vascular events like CAD which directly increases the morbidity \& smortality and PAD, which may hamper the recovery of limb movements. Peripheral Arterial Disease:-Ischemic occlusion, mediated by atherosclerosis or acute embolisation of peripheral vessels manifests as features of PAD- pain, pallor, paresthesia, pulselessness etc. Ankle-Brachial Index:-ABI is the ratio of systolic blood pressure in lower limb to systolic BP in upper limb, taken with the help of a portable doppler probe. Normal ABI is 0.9 to 1.3. Abnormal ABI value is defined as $<0.9$ which suggests the presence of peripheral arterial disease(PAD).Many studies have been conducted which have proven a role of abnormal ABI as predictor of future athero-thrombotic events such as CAD,PAD,CVD ${ }^{[2,3,4]}$ Further studies have shown to have correlation between low $\mathrm{ABI}$ and ischemic stroke ${ }^{[5,6,7,8]}$ It has also been used as a prognostic marker in stroke patients predicting increase in all-cause mortality and morbidity related to cardiovascular \& other major vascular events. ${ }^{\left[9{ }^{10]}\right.}$ Further low $\mathrm{ABI}$ as indicator of asymptomatic PAD is associated with worse limb function, possibly hampering post-stroke recovery. The proper management of PAD can improve stroke rehabilitation. ${ }^{[11]}$ As the other tools or methods, which can be used to indicate these vascular involvements like angiography etc. are either invasive or economically not feasible, ABI has emerged to be an easy screening tool for risk assessment of these events. Hence we planned to carry out a study of ankle brachial index in stroke patients. 


\section{Materials And Method}

A prospective observational study was carried out in patients presenting with stroke to the IPD of General Medicine Department of Dr S N Medical College and Associated Hospitals over a period of more than 6months.

Inclusion Criteria:- All patients with clinical and imaging based evidence of stroke

Stroke is defined as an abrupt onset neurological deficit, which is attributable to a vascular cause, usually diagnosed by brain imaging (CT or MRI)

\section{Exclusion Criteria:-Patients excluded are}

- Trauma, surgery or amputation involving the lower limb

- Leg ulcers

- Deep vein thrombosis

- Filariasis or lower limb swelling due to other causes which would impair Doppler image quality.

Data was statistically analyzed using the software SPSS. Statistical analysis was done using chi-square test and student-t test. Parametric data were expressed as mean value \pm standard deviation (SD) and categorical variables as percentage. The ankle-brachial index is the ratio of the systolic pressure in the ankle to the systolic pressure in the arm. We can measure the ankle-brachial index with a blood pressure cuff, sphygmomanometer, and handheld Doppler device. In our study we used Life drop model L150R hand held Doppler device. We used $5 \mathrm{MHz}$ and $8 \mathrm{MHz}$ transducer probe in our study to detect peripheral artery pressure.

\section{Observations \& Results}

After scrutinizing 58 patients, 50 met the criteria of inclusion, evaluated and following results were found.

1) Maximum patients (14/50) were in 66-75yrs age group. Mean age of females was higher than of males.

2) Among co-morbid conditions, HTN was the most common (46.66\%) followed by DM, past CVA, CAD. A significant proportion $(24 \%)$ had recurrent stroke.

3) WHR was high in $74.3 \%$ of all males and $66.6 \%$ of all females.

4) $24 \%$ were smokers and $32 \%$ were tobacco chewers.

5) Mean systolic BP was significantly different in upper \& lower limb on each side as shown in table 1. (P $<0.001)$.

Table 1: Mean systolic BP in all 4 limbs

\begin{tabular}{|l|l|l|l|}
\hline Systolic BP & $\begin{array}{l}\text { Upper Limb } \\
\text { Mean } \pm \text { SD }\end{array}$ & $\begin{array}{l}\text { Lower Limb } \\
\text { Mean } \pm \text { SD }\end{array}$ & P value \\
\hline RT & $154.84 \pm 26.46$ & $122.64 \pm 40.48$ & $<0.0001$ \\
\hline LT & $158.70 \pm 26.01$ & $127.30 \pm 45.80$ & $<0.0001$ \\
\hline
\end{tabular}

6) In our study population $78 \%$ had ischemic and $14 \%$ had hemorrhagic stroke, while $8 \%$ had mixed ischemic + hemorrhagic stroke.

7) Among 50 patients, $42 \%$ had low $\mathrm{ABI}$ in the range of moderate PVD. (0.4-0.7) shown in figure 1.

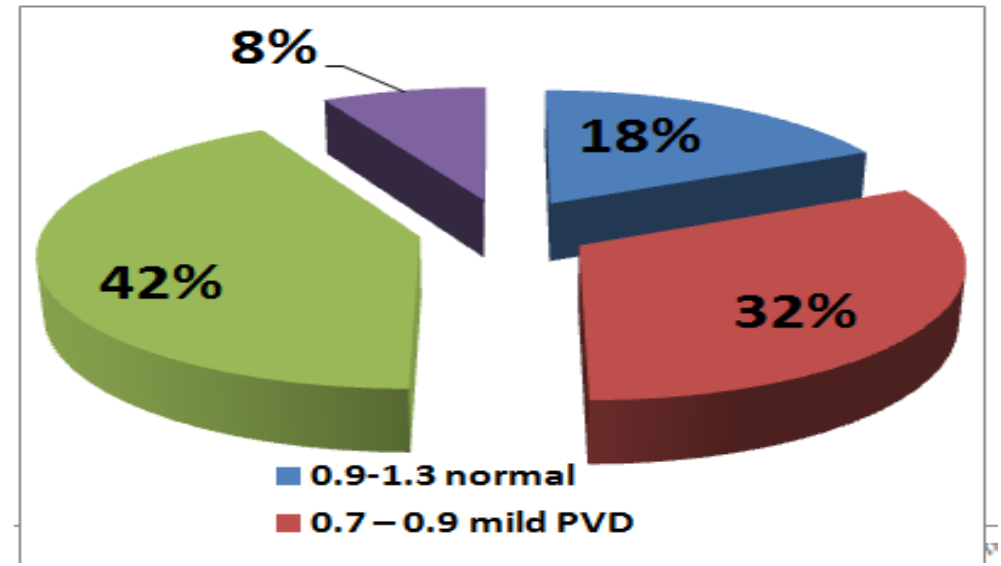

Fig.1: ABI Distribution 
8) A statistically significant correlation was found between ischemic type of stroke and ABI. ( $<<0.001)$ (Table 4) (Figure 2)

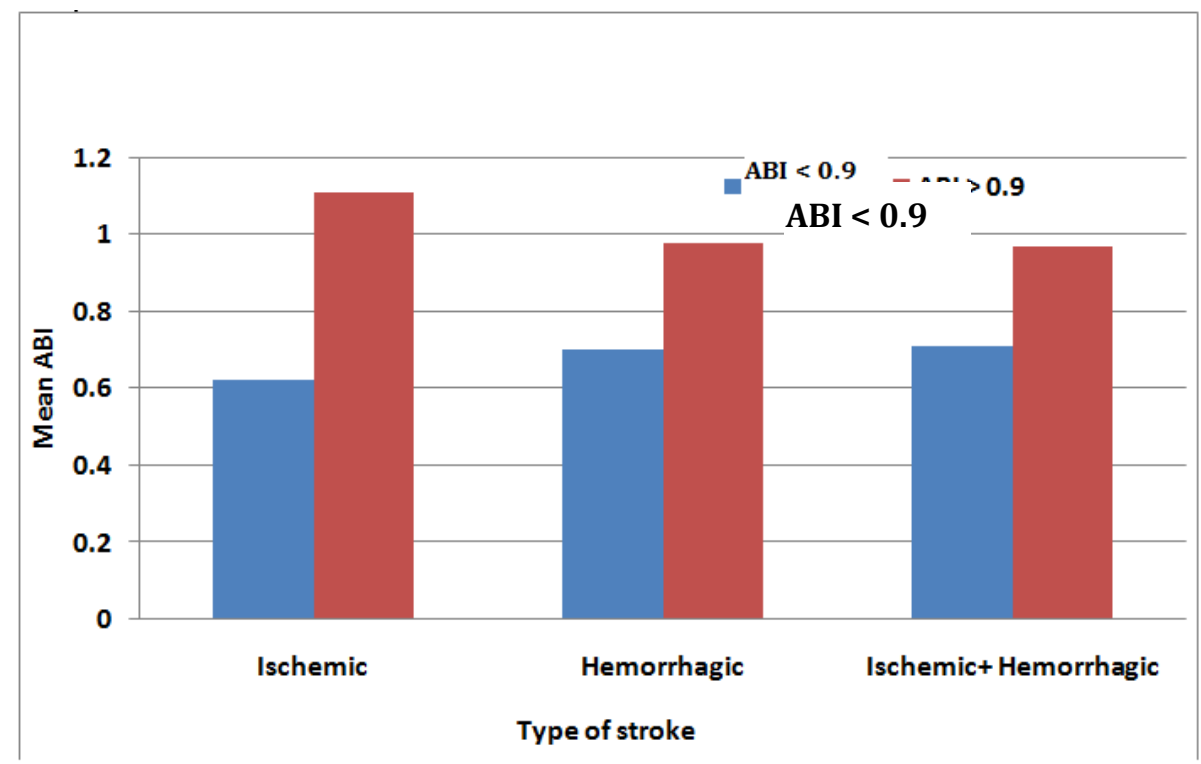

Fig.2: Type of stroke and mean ABI

9) Patients with abnormal WHR were significantly higher in number in low ABI group. ( $\mathrm{P}<0.0001)$ but mean WHR didn't show significant correlation with ABI. 10) a)The no. of patients with high systolic BP $(>140 \mathrm{mmHg})$ was significantly higher in low ABI group. $(\mathrm{P}<0.001) .($ table 2$)$

Table 2: Systolic BP of patients and ABI (upper Limb)

\begin{tabular}{|c|c|c|c|c|}
\hline $\begin{array}{c}\text { Systolic BP } \\
\text { Upper Limb }\end{array}$ & Number & ABI $<0.9$ & ABI $>0.9$ & P value \\
\hline $141-160$ & 14 & 13 & 01 & 0.001 \\
\hline$>160$ & 27 & 22 & 05 & 0.001 \\
\hline Total & 41 & 35 & 6 & 0.000 \\
\hline
\end{tabular}

b) $\mathrm{ABI}$ was found to be significantly correlated with difference in systolic BP of upper \& lower limb at each side. Mean of difference was significantly higher in low ABI group. (P value $\mathbf{0 . 0 3}$ at Lt. \& $\mathbf{0 . 0 2}$ at Rt.)Systolic BP in each lower limb was significantly different in low \& normal ABI group (P value $<0.0001$ )

Table.3: Mean systolic BP in low \& normal ABI patients

\begin{tabular}{|l|l|l|l|}
\hline SYSTOLICBP & $\begin{array}{l}\text { ABI }<\mathbf{0 . 9} \\
(\mathbf{n = 4 1})\end{array}$ & $\begin{array}{l}\text { ABI }>\mathbf{0 . 9} \\
(\mathbf{n = 9})\end{array}$ & P value \\
\hline Upper Limb RT & $154.92 \pm 27.64$ & $154.44 \pm 21.61$ & 0.961 \\
\hline Upper Limb LT & $158.60 \pm 25.80$ & $159.11 \pm 28.55$ & 0.959 \\
\hline Lower Limb RT & $111.46 \pm 32.37$ & $173.56 \pm 35.20$ & $<\mathbf{0 . 0 0 0 1}$ \\
\hline Lower Limb LT & $116.32 \pm 40.82$ & $177.33 \pm 32.88$ & $<\mathbf{0 . 0 0 0 1}$ \\
\hline RT-RT (upper/lower) & $43.95 \pm 26.44$ & $21.33 \pm 19.90$ & $\mathbf{0 . 0 2 0}$ \\
\hline LT-LT(upper/lower) & $45.32 \pm 31.55$ & $21.33 \pm 15.94$ & $\mathbf{0 . 0 3 2}$ \\
\hline Upper limb (RT-LT) & $17.15 \pm 13.90$ & $8.22 \pm 6.44$ & 0.067 \\
\hline Lower Limb (RT-LT) & $12.66 \pm 10.33$ & $4.67 \pm 6.08$ & $\mathbf{0 . 0 3 1}$ \\
\hline
\end{tabular}




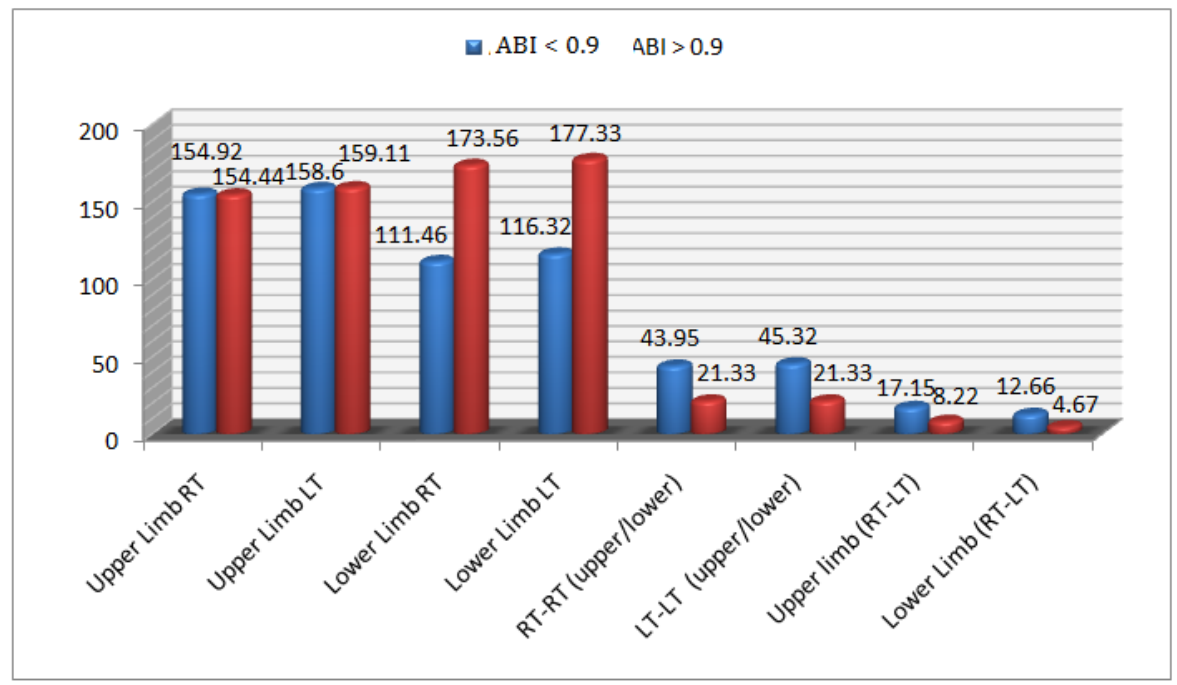

fig.3 Mean systolic BP in low \& normal ABI patients

c) Out of 50 stroke patients $46 \%$ (23/50)had difference of $>10 \mathrm{mmHg}$ in SBP of both upper limbs and 22 of them had low ABI. Although the correlation was not statistically significant, yet it was clinically relevant as it shows the severity of atherosclerosis.

11) Number of patients with co-morbid conditions was higher in low $A B I$ group but no statistical correlation could be established between $\mathrm{ABI}$ and any co-morbidity.

12) Patients with high blood sugar and dyslipidemia were significantly higher in no. in low ABI group but the mean values had no statistical correlation with ABI.

Table 4: Overview of risk factors and other clinical parameters in stroke patients

\begin{tabular}{|l|l|l|l|l|}
\hline Parameters & All patients & ABI $<0.9$ & ABI $>0.9$ & P value \\
\hline Mean age (in years) & $62.68 \pm 15.04$ & $62.92 \pm 14.15$ & $61.56 \pm 19.56$ & 0.807 \\
\hline Male/ Female & $35 / 15$ & $27 / 14$ & $8 / 1$ & 0.247 \\
\hline Urban/ Rural & $36 / 14$ & $30 / 11$ & $6 / 3$ & 0.697 \\
\hline Hypertension & 17 & 14 & 03 & 0.963 \\
\hline Diabetes & 25 & 22 & 03 & 0.269 \\
\hline IHD & 08 & 08 & 00 & 0.148 \\
\hline Past CVA & 12 & 10 & 02 & 0.890 \\
\hline Smoking & 13 & 11 & 02 & $\mathbf{0 1 3}$ \\
\hline Tobacco & 16 & 14 & 02 & $\mathbf{0 . 0 0 3}$ \\
\hline Alcohol & 05 & 04 & 01 & 0.180 \\
\hline Opium & 02 & 01 & 01 & 1.000 \\
\hline Hyperlipidemia & 21 & 18 & 03 & $\mathbf{0 . 0 0 1}$ \\
\hline Waist Hip Ratio & $0.952 \pm .12$ & $0.947 \pm .13$ & $0.977 \pm .10$ & 0.512 \\
\hline $\begin{array}{l}\text { Systolic BP } \\
\text { limb (RT-LT) }\end{array}$ & $164.54 \pm 25.55$ & $165.34 \pm 25.47$ & $160.89 \pm 27.15$ & 0.641 \\
\hline $\begin{array}{l}\text { Upper } \\
\text { difference }\end{array}$ & & $17.15 \pm 13.90$ & $8.22 \pm 6.44$ & 0.067 \\
\hline $\begin{array}{l}\text { Lower Limb } \\
\text { ABfference }\end{array}$ & $0.721 \pm .227$ & $0.645 \pm .17$ & $1.07 \pm .13$ & $<\mathbf{0 0 0 0 1}$ \\
\hline $\begin{array}{l}\text { Ischemic stroke } \\
\text { Hemorrhagic stroke }\end{array}$ & 07 & 33 & $06(1.11 \pm 0.14)$ & $<\mathbf{0 . 0 0 0 1}$ \\
\hline
\end{tabular}

\section{Discussion}

Atherosclerosis, being a generalized process affects all major vascular territories as coronary \& cerebral vessels, peripheral vessels are no exception. CAD, CVD being the field of major concern now-a-days, PAD still remains a neglected part. In our previous study we had shown a correlation between asymptomatic CAD and PAD in 
type-2 DM patients ${ }^{[14]}$. Prevalence of Peripheral Arterial Disease (PAD) in stroke patients plays an important role in secondary prevention \& rehabilitation. Early detection of PAD with easy, non-invasive and economic tool -ABI, can help to take necessary steps to facilitate functional recovery \& to prevent complications related to atherosclerosis and thus to decrease all cause morbidity \& mortality in stroke patients. Hence we carried out a study of Ankle- Brachial Index in stroke patients.

A total 50 consecutive patients of cerebrovascular stroke, who were admitted in our hospital, were included. Their risk factors (h/o DM, HTN, IHD, past CVA) and other clinical parameters (WHR, Systolic BP) were studied and ABI was measured. All these parameters were compared in low \& normal ABI group.

Mean age of patients was 62.68 \pm 15.04 year. Among risk factors, WHR was high in majority (72\%), HTN was the most common co-morbid condition, $24 \%$ had recurrence of stroke. Tobacco addiction was the most common in addiction habits.

Systolic BP was significantly different in upper \& lower limb at each side $(\mathrm{P}<0.0001)$.

Majority of patients (39/50) had ischemic stroke. Mean ABI was $0.72 \pm 0.227$ and most of the patients had low $\mathrm{ABI}$ in range of moderate $\mathrm{PVD}(0.4-0.7)$. We found low $\mathrm{ABI}$ in $82 \%$ of patients, Weimer et al[6]found $\mathrm{ABI}<0.9$ in 52.8\% of his ischemic stroke patients. Prevalence of low ABI was $40.5 \%$ in a study byAlvarez_Sabin et $\mathrm{al}^{[12]}$ where mean ABI was $0.92 \pm 0.21$. Patients with high SBP were more in low ABI group. Although no statistical correlation was found between SBP and ABI, similar with Chung et al. ${ }^{[7]}$, Chun Yi-Li et al ${ }^{[13]}$ Systolic BP in lower limb was significantly different in low \& normal ABI group.(P value $<0.0001)$.A difference of $>10 \mathrm{mmHg}$ between SBP of both upper limbs was found in $23 / 50$ patients, majority (22/23) of which had low ABI. It was found as an important marker of severity of atherosclerosis. A statistically significant correlation was found between ABI and ischemic stroke $(\mathrm{P}<0.001)$. Similarly Chun Yi-Li et al. ${ }^{[13]}$ found a strong correlation between ABI and ischemic stroke (P value $<0.008$ )where the prevalence of low ABI was $25.9 \%$.

Low ABI was associated with recurrence of atherosclerotic major vascular events (IHD, recurrent CVA).Weimer et al ${ }^{[6]}$ also found that Patients with an $\mathrm{ABI}<0.9$ had a significantly higher risk of recurrent stroke or cardiovascular death and a higher recurrent stroke risk than patients with $\mathrm{ABI}>0.9$. Alvarez-Sabin et $\mathrm{al}^{[12]}$ found that patients of stroke with low ABI had more vascular events (27\% Vs. 14\%). Patients with dyslipidemia and high WHR were more common in low ABI group but no correlation was found between mean values of lipid profile, WHR and ABI. Chung et al ${ }^{[7]}$ also found that dyslipidemia was more common in patients with low ABI, but mean levels of blood sugar, all parameters of lipid profile didn't show any correlation with ABI. Chun Yi-Li et al. ${ }^{[13]}$ also did not find a significant correlation between $\mathrm{ABI}$ and mean levels of lipid profile.

\section{Conclusion}

This study is an attempt to highlight the universality of atherosclerotic process involving all blood vessels in the body. Patients with PAD are three times more likely to develop stroke. Despite this well established fact, PAD remains understudied and often under-rated as a risk factor for stroke. In our study, a statistically significant correlation was found between ABI and stroke, more with ischemic type with major burden of PAD (41/50) as low ABI. A significant proportion of patients had previous stroke and IHD.A clinically relevant difference of $>10 \mathrm{mmHg}$ in SBP of both upper limbs was found, showing the burden of atherosclerosis. In our previous study in asymptomatic DM-2 patients, a good correlation was also found between ABI \& CAD ${ }^{[14]}$. Although the number of patients was small in our study, statistically significant values linking stroke \& ABI was found. It needs to be further evaluated in a detailed multicentric prospective study.sss

\section{References}

[1]. Fauci AS, Kasper DS, Longo DL, Braunwald E, Hauser SL, Jameson JL,et al. Harrison's Principles of internal medicine. United State; $18^{\text {th }}$ edition 2012)

[2]. Banerjee A, Fowkes F, Rothwell P. Associations Between Peripheral Artery Disease and Ischemic Stroke: Implications for Primary and Secondary Prevention. Stroke. 2010;41(9):2102-2107.

[3]. Agnelli G, Cimminiello C, Meneghetti G, Urbinati S. Polyvascular Atherothrombosis Observational Survey (PATHOS) Investigators. Low ankle-brachial index predicts an adverse 1-year outcome after acute coronary and cerebrovascular events. J Thromb Haemost. 2006;4: 2599-2606.

[4]. Busch M, Lutz K, Rohl J, Neuner B, Masuhr F. Low Ankle-Brachial Index Predicts Cardiovascular Risk After Acute Ischemic Stroke or Transient Ischemic Attack. Stroke. 2009;40(12):3700-3705.

[5]. Topakian R, Nanz S, Rohrbacher B, et al. High prevalence of peripheral arterialdisease in patientswith acute ischaemicstroke. Cerebrovasc Dis 2010;29:248-54

[6]. WeimarC, GoertlerM, Rother J, et al. Systemicriskscore evalua- tion in ischemic stroke patients (SCALA): a prospective cross sectional study in 85 German stroke units. J Neurol2007;254: 1562-08.

[7]. Chung, P., Kim, D., Kim, H., Park, K., Park, T., Hong, J., Kim, G., Bang, O., Oh, K. and Lee, S. Differences of Ankle-Brachial Index according to Ischemic Stroke Subtypes: The Peripheral Artery Disease in Korean Patients with Ischemic Stroke (PIPE) Study. Eur 
Neurol,(2013) 69(3), pp.179-184.

[8]. Braunwald E, Zipes D, Libby P. Heart disease. Philadelphia: Saunders; 2001.

[9]. Newman, A., Shemanski, L., Manolio, T., Cushman, M., Mittelmark, M., Polak, J., Powe, N. and Siscovick, D. (1999). Ankle-Arm Index as a Predictor of Cardiovascular Disease and Mortality in the Cardiovascular Health Study.Arteriosclerosis, Thrombosis, and Vascular Biology, (1999)19(3), pp.538-545.

[10]. Polisetty, S. and Veni Avvaru, D. Assessment of Ankle Brachial Index among Stroke and Nonstroke patients in a Tertiary care hospital.IOSRJDMS,(2014) 13(12), pp.01-03.

[11]. McDermott MM, Guralnik JM, Tian L, Liu K, Ferrucci L, Liao Y, Sharma L, Criqui MH. Associations of borderline and low normal anklebrachial index values with functional decline at 5-year follow-up: the WALCS (Walking and Leg Circulation Study). J Am Coll Cardiol.2009;53:1056-1062. .

[12]. Alvarez-Sabín, J. et al. "Low Ankle-Brachial Index Predicts New Vascular Events And Functional Outcome After 1 Year In Patients With Non-Cardioembolic Stroke: Our Experience And Review". Eur J Neurol 21.1 (2013): 100-106. Web.

[13]. Yi Li Chun, Hsin Fan Wang, and Shu Yi Chen. "High Risk For Future events In Acute Stroke Patients With AN Ankle Brachial Index Less Than 0.9" Acta Carrdiol Sin28 (2012): 17-24.

[14]. Kailash Babu Garg, Priyanka.P, Alok Gupta, Sandeep Tak, Neha Sharma, Rajkumar Sehra. A Study of Ankle Brachial Index And Asymptomatic Coronary Artery Disease in Type-2 Diabetes Mellitus Patients. IOSR Journal of Dental and Medical Sciences (IOSRJDMS Volume 15, Issue 8 Ver. IX (August. 2016), PP 48-51.www.iosrjournals.org.

\begin{tabular}{lll}
\multicolumn{3}{l}{ Abbreviations:- } \\
ABI $\quad:$ & Ankle- Brachial Indexl \\
PAD & $:$ & Peripheral Arterial Disease \\
IHD & $:$ & Ischemic Heart Disease \\
HTN & $:$ & Hypertension \\
DM & $:$ & Diabetes Mellitus
\end{tabular}

